\title{
How I manage acute lymphoblastic leukemia in fanconi anemia?
}

\section{Introduction}

Fanconi anemia (FA) is a genomic instability situation characterized by a wide array of congenital anomalities, bone marrow failure, leads to malignancy. The cumulative incidence of leukemia in Fanconi anemia is about $10 \%$ until first 25 years of age, and the cumulative incidence of any hematological dyscrasia is up to $90 \%$. In this disorder $94 \%$ of leukemias are myeloid as compared to $84 \%$ of leukemia's being lymphoid in non-Fanconic patients. ${ }^{1}$ This association suggests that FA is a preleukemic condition for acute myeloid leukemia (AML). Acute lymphoblastic leukemia (ALL) has also rarely been reported.

\section{Body of article}

Because of their rarity, there is no consensus on how to treat Acute Lymphoblastic leukemias when they develop. Reports of immune function in this population are limited but, these patients have a degree of primary immunodeficiency; absolute numbers of B cells and natural killer (NK) cells were reduced compared to controls, while absolute number of $\mathrm{T}$ cells were reported to be within normal range. ${ }^{2}$ Perforin and enzyme content of NK cells was reduced along with the NK cell cytotoxicity. Proliferation in response to tetanus was decreased while responses to phytohemagglutinin and candida were not. Cytotoxic $\mathrm{T}$ cell function was also reduced. Immunoglobulin $\mathrm{G}$ levels were normal in those who were evaluated. ${ }^{3}$ Antibiotic chemoprophylaxis has a major complementary role in patients with persistent infections despite "optimal" immunoglobulin replacement (residual IgG concentration $>8 \mathrm{~g} / \mathrm{L}$ ), with same modalities as in agammaglobulinemia. ${ }^{4}$ So, co-administration of IVIG $0.4 \mathrm{gr} / \mathrm{kg}$ with antibiotic and antifungal prophylaxis for primary immune deficiency disorders; cotrimoxazole for cellular immune deficiency at start of chemotherapy regimen is suggested. ${ }^{3}$ If FA patient live in developing countries and has probable malnutrition WHO guidelines for the management of children with severe acute malnutrition are also recommended.

Box 1: If the child has no complication,: cotrimoxazole for 5 days (20mg of sulfamethoxazole $+4 \mathrm{mg}$ of trimethoprim per kilogram orally twice daily) is prescribed. If child has complication: ampicillin (25-50mg/kg IM/IV 6-hourly for 2days), then oral amoxicillin (15mg/ kg 8-hourly for 5days) OR, if amoxicillin is not available, oral Ampicillin (25-50mg/kg 6-hourly for 5days) over a total of 7days and Gentamicin $(7.5 \mathrm{mg} / \mathrm{kg}$ IM $/ \mathrm{IV})$ once daily for 7 days are prescribed. If the child hasn't improvement within 48 hours: chloramphenicol ( $25 \mathrm{mg} / \mathrm{kg} \mathrm{IM} / \mathrm{IV} 8$-hourly) for 5 days adds to this regimen. If other specific infections are identified (such as pneumonia, dysentery, skin or soft tissue infections): give antibiotics as appropriate. Antibiotics should be continued for at least 5 days. The duration of treatment depends on the response and nutritional status of the child. If anorexia persists after 5 days of treatment, give the child another 5-day course. The regimen should be adapted based on local resistance patterns. ${ }^{4}$ It seems FA induced acute leukemia classification as High risk or standard risk in ALL or cytogenetic based risk stratification in AML are defeasible. FA patients should be transplanted finally, but they have chromosomal breakage constitutionally, therefore in these cases
Volume 6 Issue 5 - 2018

\author{
Abdolkarimi Babak,' Shahriari Mahdi² \\ 'Department of pediatrics, Lorestan University of Medical \\ Sciences, Iran \\ ${ }^{2}$ Associate professor of Pediatric Hematology/Oncology, Shiraz \\ University of Medical Sciences, Iran
}

Correspondence: Shahriari Mahdi, Associate professor of Pediatric Hematology/Oncology, Shiraz University of Medical Sciences, Shiraz, Iran, Email shajryar@sums.ac.ir

Received: May 15, 2018 | Published: October 31, 2018

modified chemotherapy dosing with removal of DNA cross-linking agents (Cyclophosphamide) are suggested; in order to reach sustain bone marrow remission and decrease chemotherapy-related toxicities. The majority of FA patients involved by acute leukemia were treated by classic chemotherapy protocols had been died..$^{1,5,6}$ Therefore choosing treatment protocols based on routine classification should be avoided. The oncologists should suffice to non-myelosuppresive agents such as steroids, Vincristin, Asparginase in ALL patients. Children oncology group (COG) has chemotherapy regimen for lesser risk $\mathrm{ALL}^{7}$ include:

Induction phase:

a. Vincristine $1.5 \mathrm{mg} / \mathrm{m}^{2}$ IV on days $1,8,15,22$

b. Prednisone $40 \mathrm{mg} / \mathrm{m}^{2}$ in on days 1 to 28 divides 3 doses then taper in one week

c. L-asparaginase 6,000units $/ \mathrm{m}^{2} \mathrm{IM}$ on days 2, 5, 8, 12, 15 and 19

d. Intrathecal MTX on days 1, 22

Additional doses are given on days 8 and 15 when CNS status is present. In AML patients administration of routine chemotherapy protocols such as MRC12, BFM-98 include DNA cross-linking agents (Ara-C and Etoposide and antracyclines) is fatal for FA patients. $^{8}$ Traditional sight for decreasing $50 \%$ dose adjustment for these agents is unpromising. It seems that medications such as reduced dose Cytarabin should be limited to preparation for allogenic BMT if full matched donor exists. In this pre-transplant stage some modified regimens are administered such as Mini-FLAG include: Fludarabine $30 \mathrm{mg} / \mathrm{m}^{2}$ and Ara-C $300 \mathrm{mg} / \mathrm{m}^{2}$ each on days 2-4 and granulocyte-colony stimulating factor (G-CSF) $5 \mathrm{mcg} / \mathrm{kg}$ is Days 1-5 may be recommended. ${ }^{9}$ If full matched donor doesn't exist administration of metronomic chemotherapy with oral antimetabolic agents(6-Meraptopurine, 6-Thioguanine) are recommended similar to elderly patients(>65years) involved by AML .In these patients there is chromosomal breakage due to aging similar to FA patients. Therefore treatment approach in FA patients with AML is similar to elderly patients involved by AML. These patients treatment with daily oral 6-mercaptopurine $75 \mathrm{mg} / \mathrm{m}^{2}$ similar to patients with age $>60$ years, non-promyelocytic AML, the absence of uncontrolled comorbidities, and patient not amenable to curative treatment is suggested. ${ }^{10}$ Every 
time that the blast cells increases repeating Mini-FLAG regimen is suggested. Also, administration of intravenous amino-transfusion, micronutrients and non-B-vitamins including calcium, vitamins A, D, $\mathrm{C}$ and $\mathrm{E}$, fatty acids, selenium, and zinc during chemotherapy courses are useful. ${ }^{11}$

\section{Conclusion}

Because ALL patients with FA are rare, controversial topics about treatment of these patients are many.We need to many clinical trials to introduce safe and effective chemotherapy regimen which hasn't been mortality before bone marrow transplantation.

\section{Acknowledgements}

None.

\section{Conflict of interest}

There is no conflict of interest among the authors.

\section{References}

1. Shah A, John BM, Sondhi V. Acute Lymphoblastic Leukemia with Treatment-Naïve Fanconi Anemia. Indian Pediatr. 2013;50(5):508-510.

2. Orange JS. Natural killer cell deficiency. J Allergy Clin Immunol. 2013;132(3):515-526.

3. Myers KC, Bleesing JJ, Davies SM, et al. Impaired immune function in children with Fanconi anaemia. Br J Haematol. 2011;154(2):234-240.
4. Aguilar C, Malphettes M, Donadieu J, et al. Prevention of infections during primary immunodeficiency. Clin Infect Dis. 2014;15;59(10):1462-1470.

5. Marzia Lazzerini, David Tickell. Antibiotics in severely malnourished children: systematic review of efficacy, safety and pharmacokinetics. Bull World Health Organ. 2011;89(8):594-607.

6. Terrie Flatt, Kathleen Neville, Karen Lewing, et al. Successful treatment of fanconi anemia and t-cell acute lymphoblastic leukemia. Case Rep Hematol. 2012;2012:396395.

7. Sevgi Yetgin, Murat Tuncer, Ertuğul Güler, et al. Acute lymphoblastic leukemia in fanconi'sanemia. American Journal of Hematology. 1994;45(1):94-101.

8. Ibraheem Abosoudah, Taher Sumaili, Mouhab Ayas. Treatment of highrisk pre-b acute lymphoblastic leukemia in a fanconi anemia patient with reduced-intensity chemotherapy. Biol Blood Marrow Transplant. 2013;19(2):S241.

9. Mehta PA, Ileri T, Harris RE, et al. Chemotherapy for myeloid malignancy in children with fanconi anemia. Pediatr Blood Cancer. 2007;48(7):668-672.

10. Kapoor A, Beniwal SK, Kalwar A, et al. Metronomic therapy with oral 6-mercaptopurine in elderly acute myeloid leukemia: A prospective pilot study. South Asian J Cancer. 2016;5(2):70-72.

11. Evelyn Ward. Advice on Nutritional Problems for Patients with Fanconi Anaemia. Fanconi hope; 2009:1-13. 